\title{
Self-sampling for HPV to enhance uptake of cervical cancer screening: Has the time come in Canada?
}

\author{
Aisha Lofters MD PhD, Mandana Vahabi PhD RN
}

$\longrightarrow$ ervical cancer is uniquely preventable with programs of regular screening. In many high-income nations, including Canada, the incidence of and mortality associated with cervical cancer have declined steadily and steeply as a result of widespread use of the Papanicolaou (Pap) test as a screening tool. ${ }^{1}$ In Canada, the mortality for cervical cancer decreased by 2.3\% per year between 2001 and 2010, and this decline was associated with a reduction in incidence over the same period that was considered to be largely due to screening. ${ }^{2}$ The screening participation rate in Canada for 2009-2011 was $69 \% ;^{3}$ however, certain subgroups of women, including immigrants and women with low income, are less likely to be appropriately screened for cervical cancer, with correspondingly poorer outcomes. ${ }^{4}$ Screening strategies that involve self-sampling for human papillomavirus (HPV), a key causal factor in cervical cancer, may help to close this gap in Canada.

Self-sampling for HPV does not require a pelvic examination. It involves self-collection of vaginal material, via devices such as swabs, tampons or brushes, and analysis of the sample for high-risk HPV strains. ${ }^{5}$ Data from randomized trials have suggested that testing for HPV is a more appropriate and effective method of screening for cervical cancer than cytologic screening. ${ }^{6}$ A recent meta-analysis suggested that testing for high-risk HPV on self-collected vaginal samples is at least as sensitive, although not as specific, as cytologic examination of clinician-collected cervical samples. ${ }^{5}$ A growing body of evidence from randomized trials around the world shows that mailing self-sampling kits leads to increased screening participation for underscreened women relative to cliniciancollected samples. ${ }^{7}$ Although much of the literature comes from Europe, self-sampling kits have been shown to be feasible in Canadian populations, including underhoused women in British Columbia $^{8}$ and First Nations women in northwest Ontario. ${ }^{9}$ However, the studies were small and did not provide evidence to support incorporation of HPV self-sampling into any of the provincial cervical cancer screening programs.
Perhaps Canada can learn from the Finnish and Dutch examples. Both countries have explored the use of HPV self-sampling within organized screening programs for women who are overdue for screening. In Finland, all women aged 30 to 60 years are invited to participate in screening every five years. As part of a randomized trial conducted within the context of the country's routine cervical cancer screening program, 8700 women in the Finnish Cancer Registry who did not participate in screening after their first invitation were randomly assigned to receive a self-sampling kit or a reminder letter. ${ }^{10}$ Women who self-sampled and had a positive result were invited for a follow-up Pap test (age $<40$ yr) or were referred directly for colposcopy (age $\geq 40 \mathrm{yr}$ ). Participation was significantly higher among women who received the self-sampling kit $(31.5 \%, 95 \%$ confidence interval [CI] $29.7 \%-33.4 \%$ v. $25.9 \%, 95 \%$ CI $24.8 \%-27.0 \%) .{ }^{10}$ Loss to follow-up after a positive HPV result was $13.4 \%$. Self-sampling also appeared to prompt immigrant women (who had lower participation rates) more efficiently than the reminder letter. Subsequent work conducted within the Finnish screening program showed that a reminder letter followed by a mailed self-sampling kit increased participation from $72.6 \%$ to $82.2 \% .^{11}$

In the Netherlands, the PROHTECT study (Protection by Offering Human Papillomavirus Testing on Self-sampled Cervicovaginal Specimens Trial) was conducted with 28000 women within the country's population-based screening program. ${ }^{12}$ Women who did not respond to an initial screening reminder received either a second letter or a self-sampling kit, and those with

\section{- KeY POINTS}

- In Canada, cervical cancer screening continues to be underutilized among hard-to-reach women.

- A growing body of evidence supports self-sampling for human papillomavirus (HPV), the virus that causes cervical cancer, as a method to increase screening participation for these women.

- Large-scale, high-quality studies of HPV self-sampling are needed in Canadian jurisdictions to evaluate the potential effect of this innovative approach. 
a positive HPV result were instructed to visit their doctor for a follow-up Pap test. In the selfsampling group, $27.5 \%$ of women submitted a sample, whereas $16.6 \%$ of women in the recall letter group saw their provider for a Pap test. More than $90 \%$ of women with a positive HPV result in the self-sampling group complied with follow-up. As a result of this work, the Dutch screening program has now committed to selfsampling. As of 2016, women aged 30 to 60 years will be invited every five years to have a cervical specimen collected by a clinician, which will be tested for HPV. Women who do not respond will be given the opportunity to request a free self-sampling kit to be mailed to their home. Other high-income jurisdictions should closely observe the findings from this new element of the program over the next few years.

Studies of similar quality are needed in Canadian jurisdictions to rigorously evaluate the feasibility, acceptability, loss to follow-up and costeffectiveness of HPV self-sampling on a large scale, as well as to clarify important implementation considerations. For example, despite widespread positive views among women, barriers to self-sampling have been raised in the literature. A qualitative study published in 2009 found that immigrants and women of low socioeconomic status in Canada were generally receptive to selfsampling, but there were notable concerns about test accuracy and about women's ability to collect the sample properly. ${ }^{13}$ Investment in education for women regarding how to use the test, the meaning of test results and actions to be taken in the face of HPV detection is required. Opt-in strategies would likely reduce the number of wasted kits, but these strategies may not be effective in increasing screening rates. ${ }^{7}$ It would be important for self-sampling to be offered only to women who have not regularly participated in screening, as the literature does not support encouraging women to "switch" from routine office-based sampling to self-sampling. Consideration would also need to be given to which device to endorse, on the basis of price, accuracy, ease of use and other relevant factors. Finally, in Canada's three territories and the province of Quebec, which do not have organized cervical cancer screening programs, ${ }^{3}$ the effect of HPV self-sampling may be quite different, and different approaches may be required in those jurisdictions.

Self-sampling for HPV has the potential to surmount many existing barriers to cervical cancer screening and to empower women to conduct the test in a private location and at a time of their choosing. Evidence suggests that some underscreened women may prefer HPV self-sampling to traditional collection of samples by clinicians. If implemented appropriately, self-sampling could lead to increased participation in cervical cancer screening programs for hard-to-reach women and a resultant reduction in cervical cancer screening inequalities. This approach could transform existing screening paradigms. The time has come for large-scale, rigorous research in Canada to explore the feasibility of this innovative method and to set a clear path forward for policy-makers interested in reducing cancer screening gaps, improving screening at the population level and ultimately reducing the incidence of and mortality associated with cervical cancer.

\section{References}

1. Mohar A, Frias-Mendivil M. Epidemiology of cervical cancer. Cancer Invest 2000;18:584-90.

2. Canadian cancer statistics. Toronto: Canadian Cancer Society; 2015.

3. Cancer screening in Canada: an overview of screening participation for breast, cervical and colorectal cancer. Toronto: Canadian Partnership Against Cancer; 2015.

4. Lofters AK, Ng R, Lobb R. Primary care physician characteristics associated with cancer screening: a retrospective cohort study in Ontario, Canada. Cancer Med 2015;4:212-23.

5. Snijders PJ, Verhoef VM, Arbyn M, et al. High-risk HPV testing on self-sampled versus clinician-collected specimens: a review on the clinical accuracy and impact on population attendance in cervical cancer screening. Int J Cancer 2013; 132:2223-36.

6. Ronco G, Dillner J, Elfström KM, et al. Efficacy of HPV-based screening for prevention of invasive cervical cancer: follow-up of four European randomised controlled trials. Lancet 2014;383:524-32

7. Verdoodt F, Jentschke M, Hillemanns P, et al. Reaching women who do not participate in the regular cervical cancer screening programme by offering self-sampling kits: a systematic review and meta-analysis of randomised trials. Eur J Cancer 2015;51: 2375-85.

8. Ogilvie G, Krajden M, Maginley J, et al. Feasibility of selfcollection of specimens for human papillomavirus testing in hard-to-reach women. CMAJ 2007; 177:480-3.

9. Zehbe I, Moeller H, Severini A, et al. Feasibility of selfsampling and human papillomavirus testing for cervical cancer screening in First Nation women from Northwest Ontario, Canada: a pilot study. BMJ Open 2011;1:e000030.

10. Virtanen A, Nieminen P, Luostarinen T, et al. Self-sample HPV tests as an intervention for nonattendees of cervical cancer screening in Finland: a randomized trial. Cancer Epidemiol Biomarkers Prev 2011;20:1960-9.

11. Virtanen A, Anttila A, Luostarinen T, et al. Improving cervical cancer screening attendance in Finland. Int $J$ Cancer 2015;136:E677-84.

12. Gök M, Heideman DA, van Kemenade FJ, et al. HPV testing on self collected cervicovaginal lavage specimens as screening method for women who do not attend cervical screening: cohort study. BMJ 2010;340:c1040.

13. Howard M, Lytwyn A, Lohfeld L, et al. Barriers to acceptance of self-sampling for human papillomavirus across ethnolinguistic groups of women. Can J Public Health 2009;100:365-9.

Affiliations: Centre for Research on Inner City Health (Lofters), Li Ka Shing Knowledge Institute, St. Michael's Hospital, Toronto, Ont.; Department of Family and Community Medicine (Lofters), University of Toronto, Toronto, Ont.; Daphne Cockwell School of Nursing (Vahabi), Ryerson University, Toronto, Ont.

Contributors: Aisha Lofters and Mandana Vahabi contributed equally to the conception of this commentary. Aisha Lofters drafted the article, and Mandana Vahabi revised the article critically for intellectual content. Both of the authors gave final approval of the version to be published and agreed to serve as guarantors of the work. 